\title{
Théologiques
}

Théologiques

\section{Père Ie Dieu}

\section{Jean Larose}

Volume 6, numéro 1, mars 1998

Autres regards sur la laïcité

URI : https://id.erudit.org/iderudit/024955ar

DOI : https://doi.org/10.7202/024955ar

Aller au sommaire du numéro

\section{Éditeur(s)}

Faculté de théologie de l'Université de Montréal

ISSN

1188-7109 (imprimé)

1492-1413 (numérique)

Découvrir la revue

\section{Citer cet article}

Larose, J. (1998). Père Ie Dieu. Théologiques, 6(1), 55-62.

https://doi.org/10.7202/024955ar

\section{Résumé de l'article}

Ce court article renferme des questions d'un non-croyant pratiquant adressées a des catholiques pratiquants. L'auteur se demande, entre autres, pourquoi la religion chrétienne supporte aussi mal la modernité alors que cette dernière en est issue? Il propose également des réflexions visant à faire sortir le sujet croyant des structures religieuses et des mentalités sécurisées. Si la religion est lieu d'humanisation, la sainteté ne serait-elle pas d'embrasser à bras le corps tous les risques du sujet, dont ceux d'aimer et de compatir? d'utilisation que vous pouvez consulter en ligne.

https://apropos.erudit.org/fr/usagers/politique-dutilisation/ 


\title{
Père le Dieu
}

\author{
Jean LAROSE \\ Professeur, Département d'études françaises \\ Université de Montréal
}

\section{RÉSUMÉ}

Ce court article renferme des questions d'un non-croyant pratiquant adressées a des catholiques pratiquants. L'auteur se demande, entre autres, pourquoi la religion chrétienne supporte aussi mal la modernité alors que cette dernière en est issue? Il propose éga lement des réflexions visant à faire sortir le sujet croyant des structures religieuses et des mentalités sécurisées. Si la religion est lieu d'humanisation, la sainteté ne seraitelle pas d'embrasser à bras le corps tous les risques du sujet, dont ceux d'aimer et de compatir?

This short article addresses the questions raised by a practicing non-believer directed at practicing Catholics. The author asks why the Christian religion barely tolerates modernity while the latter arises from it. The author equally proposes certain reflections with a view to bringing out the believing subject from religious structures and from securing mentalities. If religion is the place for humanization, then will not holiness be the embracing of all the risks of the subject, like love and compassion?

Mécréant attaché au catholicisme et qui en pratique les textes (je suis en quelque sorte un non-croyant pratiquant), je m'étonne du sort que fait à l'Église l'opinion des pays de tradition catholique. Les médias peuvent ridiculiser et même insulter le catholicisme et ses représentants, les intégristes ou les mafieux de partout assassiner des catholiques sans que cela bouleverse. Une bombe qui éclate dans une mosquée ou une synagogue déchire aussitôt notre conscience anticolonialiste ou réveille l'horreur de l'antisémitisme; mais rien de tel ne se gendarme en nous quand on tue des moines en Algérie ou des prêtres ouvriers au Brésil. Si Timor n'était pas catholique, son invasion par l'Indonésie et la répression terrible qui s'est abattue sur la population aurait ému davantage les pays riches; entre les chrétiens et les musulmans du Liban, les médias occidentaux ont désigné 
comme tout naturellement les seconds comme les victimes des premiers. Il semble que chacun dans nos pays doive un jour en passer par le rejet de la religion de ses pères, et que cela lui fera estimer que les persécutions anticatholiques sont d'une certaine manière (ce « d'une certaine manière " est peut-être le sujet de mon article) justes et méritées. Cette répulsion pour le culte qui a fait notre civilisation apparaîtra d'autant plus naturelle que la cible visée sera plus proche du centre, c'est-à-dire de l'autorité catholique romaine. Il n'est jusqu'aux catholiques pratiquants que je rencontre qui me semblent étonnamment tièdes : la dernière fois que je suis allé à la messe, j'ai eu envie de demander au prédicateur de se taire et de me laisser parler, tellement m'attristait la platitude de son commentaire des Écritures. Quand j'entends le cardinal Turcotte à la radio, il me semble toujours qu'il s'inquiète beaucoup plus de ne pas être pris pour une grenouille de bénitier que de témoigner de sa foi; tout son discours me paraît précédé d'une demande d'absolution préalable de sa position de représentant de l'autorité catholique romaine.

Pourquoi la religion chrétienne supporte-t-elle si mal la modernité? C'est bien sûr poser la question à l'envers : puisque la modernité est sortie du christianisme, c'est au christianisme qu'il faut demander ce qui lui a rendu si adverse la modernité de la civilisation qu'il a façonnée. Contenait-il les principes de la doxa antiautoritaire qui emporte notre culture? Les réponses qu'on peut fournir à cette question indiquent d'ailleurs que la crise radicale de modernité n'est peut-être qu'une question de temps pour les autres religions monothéistes: on sait bien que quand l'Islam croit résister à l'Occident en s'enivrant d'appels à la guerre sainte et en revoilant ses femmes, il ne fait que se crisper contre la modernité dont l'impérialisme fut historiquement pour les peuples musulmans l'annonciateur arrogant. Les mosquées débordantes et les écoles rabbiniques florissantes, dans le contexte politique du revival identitaire où elles s'inscrivent, sont aussi des symptômes de la crise moderne. Pourquoi ai-je l'impression que celle-ci évoluera autrement, ultimement pire pour l'Islam, bénigne pour le judaïsme, que celle qui retourne contre le christianisme en ennemis ou en indifférents hostiles les sociétés de vieille culture chrétienne.

Chacune des phrases que j'aligne exigerait déjà tant de réserves et de précisions... Je ferai pire, je brasserai naïvement - et trop lestement l'idée que si le christianisme, et particulièrement le catholicisme, souffre plus de la modernité que les autres grandes religions, c'est que cette religion est une affaire de famille. Les savants pourront me reprocher de faire un usage anhistoriciste des textes que je citerai; d'avance je confesse que je lis souvent les textes littéraires comme les croyants lisent les Écritures, en essayant de les entendre. 
Notre Dieu est personnel, et il est notre père. Son Fils est notre frère, d'ailleurs nous sommes tous frères et sœurs en Jésus-Christ. Si nous n'avons pas officiellement de déesse Mère, celle du Sauveur menace toujours (Jean-Paul Il n'a-t-il pas récemment parlé d'elle comme de la Corédemptrice?) de reprendre la place que l'imaginaire européen n'a jamais complètement retirée à la Déesse de ses premiers cultes.

Ce lien personnel avec Dieu, et plus encore avec son Fils crucifié, devait permettre à chaque individu de prendre la forme du sujet. Ajoutons la liberté : il aura fallu moins de deux mille ans pour que, le Verbe s'étant fait chair, le christianisme accouche du sujet moderne : une volonté libre, responsable et "humaine ". L'idée d'humanité est en effet venue de là, avec celle d'égalité entre les sujets qui découle en principe de l'universalité du salut.

Relisons Malraux :

Le christianisme avait été amené à représenter maints sentiments écartés avant lui. Si l'art assyrien avait figuré des torturés, il avait été indifférent à leur souffrance. Le style des Déesses-Mères de l'Euphrate se fût mal appliqué à la Vierge. Et quel art avait dû représenter une femme devant son fils supplicié? L'immense découverte chrétienne, dans le domaine de la représentation, fut que figurer n'importe quelle femme dans le rôle de la Vierge était plus émouvant que tenter de hausser ce rôle au surhumain, par l'idéalisation et le symbole. (...) Pour la statuaire antique, la partie mobile du visage (les yeux et la bouche) compte peu; mais la statuaire chrétienne s'attache passionnément à elle. Lorsque, dans un musée, nous arrivons aux salles gothiques, il nous semble rencontrer les premiers hommes vrais. Devant notre art médiéval, l'Asiatique a l'impression d'impudeur, et bien plus vive que devant les nus de la Grèce : l'art gothique a démasqué l'homme. (...)

Le saint ne l'est ni dans la mesure où il règne sur l'humanité ni dans celle où il s'en délivre, mais dans celle où il l'approfondit ${ }^{1}$.

Approfondir l'humanité par la sainteté signifie quoi? J'entends que cela veut dire en prendre le risque, tous les risques, au lieu d'essayer de les éviter en dominant ou en fuyant sa condition de sujet. Le pape, qui

1 André MALRAUX, Psychologie de l'art : la Création artistique. Genève, Skira, 1948, pp. 64-65. 
n'en manque pas une ${ }^{2}$, vient de réaffirmer que la réincarnation est une erreur. Admirable constance! La vie est donc unique et manquable. Pas de seconde chance. Toute la noblesse du sujet humain tient dans le fait d'assumer qu'il est entièrement et uniquement ce n'importe quelle femme, ce n'importe quel homme qui, depuis le Christ, est toute l'humanité par la singularité même de son individuation. Un sujet humain, et chargé encore de la part de lui-même qui lui échappe. Celle que l'amour révèle.

Quand je relis mon sermon préféré de Bossuet, je trouve en effet que l'amour a aussi reçu du catholicisme un sens que ne pouvait lui donner aucune autre religion: "C'est ce que doit faire en nous la croix de Jésus. $\mathrm{Si}$ nous ressentons ses douleurs, le monde ne peut plus avoir de douceurs pour nous : les épines du Fils de Dieu doivent avoir arraché ses fleurs; et l'amertume qu'il nous donne à boire doit avoir rendu fade le goût des plaisirs ${ }^{3}$. "

Je crois que s'il n'y a qu'en pays chrétien que l'on se sente coupable et bouleversé des souffrances historiques des "autres" - à tel point que la destruction des Juifs d'Europe, l'extermination des Indiens d'Amérique, ou la réduction en esclavage des Africains nous sont restés sur la conscience comme des crimes qui ébranlent toutes les idées que nos pères avaient pu se faire sur la noblesse de leur civilisation et leur droit de l'imposer aux autres (suivant ce même désir de domination qui s'exprime encore sereinement chez les non-chrétiens et à quoi c'est peut-être pour pouvoir redonner libre cours que nos cultures se déchristianisent) -, c'est grâce à l'idée compassionnelle de l'amour dont le catholicisme a empreint notre civilisation. N'est-il pas prodigieux de pouvoir affirmer : "Si nous ressentons ses douleurs, le monde ne peut plus avoir de douceurs pour nous". N'est-ce pas à une telle folie qu'il faut remonter pour comprendre pourquoi le seul fait que la Shoa ait pu avoir lieu transforme pour la conscience sérieuse le monde en désert? Le concept de crime contre l'humanité aurait-il pu être inventé dans une culture qui n'aurait pas reçu en modèle de l'amour le supplice volontaire de Jésus et l'affliction de la Vierge devant son fils crucifié? On voit bien que les abominations commises en Chine par les Japonais n'ont pas pris dans leur conscience historique la place d'anus mundi qu'Auschwitz occupe dans la nôtre.

2 Sur l'infaillibilité pontificale en tant qu'idée sacrilège pour l'esprit de notre temps, voir : Philippe MURAY, Le XIX siècle à travers les âges. Paris, Denoël (L'infini), 1984.

3 Bossuet, "Premier sermon pour le vendredi de la semaine de la passion, Sur la compassion de la sainte Vierge », 1660, in Sermons, Paris, Garnier, Tome, II, 1929, p. 629. 
Quand Baudelaire écrit, pour décrire son angoisse à la vue d'un misérable : « Je sentis ma gorge serrée par la main terrible de l'hystérie », il me semble que cet amour pour un pauvre - inconnu de lui, mais qui lui ressemble - est plus que de l'amour, que c'est une compassion sublime et maladive dont la possibilité remonte aux méditations sur les Plaies de Notre Seigneur. L'hystérie, cette maladie de l'identification au désir de l'autre, eut-elle inspiré la psychanalyse et la théorie des pulsions (même si ce fut à un Juif) dans une civilisation non chrétienne? Faute de meilleur mot, j'emploie ici le nom de cette maladie pour désigner, à la manière de certaines théoriciennes de "l'écriture féminine ", la mobilité libidinale, la pluralité subjective qu'elle révèle, en négatif, jusque dans la laideur et parfois l'insupportable violence de ses symptômes. On sait depuis Freud que ceux-ci sont l'expression ambivalente d'une révolte contre toute loi, pouvoir, virilité ou paternité qui voudrait réduire la féminité du sujet à de la passivité. Révolte encore, ajouterais-je, contre l'aliénation compassionnelle qui submerge le sujet, spasme contre cet amour d'identification qui lui impose de se mettre sans réserve à la place - exactement dans la peau de toute victime dans une scène d'oppression. La mélancolie, qui accompagne l'hystérie chez tant de nos grands artistes ou philosophes, eut-elle donné ces œuvres magnifiques si le christianisme n'avait inscrit le sentiment religieux dans une économie libidinale analogue à celle qui lie chaque sujet aux personnes réelles de ses père et mère? Il me semble que c'est ce mélange insupportable d'amour et de loi situant pour le sujet la personne de Dieu comme un autre sujet appréhendable par le désir, qui alimente dans les sociétés occidentales (depuis toujours, mais sous des formes qui ne cessent d'évoluer) ce qu'on pourrait appeler l'inconscient de la laïcité.

L'étreinte qui noue la Vierge et son fils crucifié incarne pour moi le génie de la religion catholique pour les formes de l'amour qui sont le plus cruelles, mais aussi le plus révélatrices de l'humanité de la vie. Le christianisme fait mal, le catholicisme plus encore, parce qu'il ne cesse de rappeler que l'humanité est une condition intenable.

Le Père et le Fils partagent dans l'éternité une même gloire, la Mère et le Fils partagent dans le temps les mêmes souffrances; le Père et le Fils une même source de plaisirs, la Mère et le Fils un même torrent d'amertume; le Père et le Fils un même trône, la Mère et le Fils une même croix. Si on perce sa tête d'épines, Marie est déchirée de toutes leurs pointes; si on lui présente du fiel et du vinaigre, Marie en boit toute l'amertume; si on étend son corps sur une croix, Marie en souffre toute la violence. (...) Cet amour fait un poids de fer sur sa poitrine, qui la serre et l'oppresse si violemment, qu'il y étouffe jusqu'aux sanglots (...) Mais surtout cet amour est un poids, parce qu'il 
pèse sur Jésus-Christ même : car Jésus n'est pas le seul, en cette rencontre, qui fasse sentir ses douleurs. Marie est contrainte malheureusement de le faire souffrir à son tour: ils se percent tous deux de coups mutuels : il est de ce fils et de cette mère comme de deux miroirs opposés, qui, se renvoyant réciproquement tout ce qu'ils reçoivent par une espèce d'émulation, multiplient les objets jusqu'à l'infini. Ainsi leur douleur s'accroît sans mesure, pendant que les flots qu'elle élève se repoussent les uns sur les autres par un flux et reflux continuel: si bien que l'amour de la sainte Vierge est en cela plus infortuné, qu'il compatit avec Jésus-Christ et ne le console pas, qu'il partage avec lui ses douleurs et ne les diminue pas: au contraire il se voit forcé de redoubler les peines du Fils, en les communiquant à la Mère ${ }^{4}$.

À quelques changements de mots près, ce texte conviendrait pour décrire les "deux miroirs opposés" qui renvoient le plaisir entre deux êtres faisant l'amour au sein de la plus vive passion. Ou encore la dynamique névrotique qui entraîne désespérément une famille, ou un couple pris dans une "folie à deux». De telles lectures seraient évidemment aussi réductrices, on peut dire aussi bête que celle qui consiste à déchiffrer comme une scène sexuelle, comme une scène dont la sexualité révèle le "vrai sens", la vision de l'ange aux dards qui ravit sainte Thérèse. Mais que ces lectures soient possibles, qu'elles puissent se présenter à l'esprit tient au fait que le christianisme est si humain par la compassion jusqu'à laquelle il a poussé l'amour, qu'il en parait analogue à la névrose hystérique. Le christianisme recèle un savoir immense sur l'amour, mais un rien, un léger déplacement de point de vue obtenu par la suspension temporaire et furtive de la foi, y révèle aussitôt que les liens qui nouent les êtres mutuellement attachés par des sentiments passionnés sont toujours susceptibles de régresser à l'enchaînement haineux. L'amour est de la haine surmontée, dira Freud. Tout le fond splendide et déchirant des névroses est là, inhérent à ce "plus grand amour" qui, dans le christianisme, consiste à « donner sa vie pour ceux qu'on aime». Cet amour suppose précisément un surmontement proche du refoulement qui est devenu moralement inacceptable pour l'hédonisme moderne, par l'effet de la mauvaise lecture de Freud qui dégrade la liaison amoureuse de la famille en pacte de refoulement au service de la Loi. Dieu le Père règne en effet sur cette scène d'amour entre le Fils couvert de plaies et sa mère infiniment en larmes. Comment son trône eut-il échappé à la révolution qui a détruit les autres monarchies? Aujourd'hui, nous ne trouvons rien si révoltant que l'idée de monarchie de droit divin : Louis XIV incarne dans la 
mémoire ce qu'il y a de plus intolérable dans la puissance royale, absolutiste, paternelle, solaire et soi-disant divine. Peut-on pourtant séparer de cet absolutisme intolérable l'amour épouvantable que décrit Bossuet? $\mathrm{Ne}$ faut-il pas un père extrême pour que soit révélée à un sujet toute son humanité? Non, cette idée est inacceptable.

Intolérable comme le péché originel. Le dogme läque d'aujourd'hui est négatif, c'est l'inexistence du péché originel associé dans les esprits au péché de la chair. On en peut déduire que la haine panique qui s'acharne contre le refoulement de la sexualité à quoi on insiste pour réduire le catholicisme ${ }^{5}$ est indirectement une haine de la compassion. C'est-à-dire un désir de se libérer de ce qui dans l'amour chrétien permet à chacun de se reconnaître sans réserve dans l'humanité des autres.

Toutes les cultures connaissent l'hystérie. Mais l'Occident catholique est seul à l'avoir dans sa religion fondu avec l'amour pour conférer à celuici, comme à l'humanité, un sens absolu. Cet amour et cette humanité cachent nécessairement un envers méconnu, qui appartient au diable. Les théologiens qui croient au Diable disent que le mal dans le monde n'est pas chaotique, qu'il est pervers. Le dyabolos n'est-il pas d'ailleurs l'ennemi du symbolon, c'est-à-dire celui qui, dans le but pervers de rendre l'histoire insensée, sème la discorde entre les hommes et leurs symboles? Sous le règne de ce qu'on pourrait appeler le règne du dyssymbole, la conscience moderne nage dans une telle détresse qu'il n'y a plus guère que "la nouvelle Isis " du rêve de Gérard de Nerval pour apaiser, si peu d'ailleurs, l'angoisse que Dieu le Père, sa loi mêlée d'amour, son amour assorti de conditions, n'arrive plus à chasser. Oui, au fond Isis est la Déesse du Nouvel Âge, nouveau polythéisme absolument sans dogme, auquel nos contemporains se convertissent en masse.

Assez d'imprudences, je m'arrête, mais j'ajoute que nous en arrivons bientôt au point où notre société sera sommée par le libéralisme de se délaïciser, si je puis dire, après s'être déchristianisée. D'ailleurs, je ne sais pas pourquoi je parle au futur, nous n'en sommes déjà plus à nous demander ce que notre société devrait garder des valeurs chrétiennes après le christianisme; on se demandera "comment était-ce quand il y avait encore des gens soucieux de l'humanité des autres? » Une secte, c'est la religion moins l'humanisme et la raison. Dans une secte, l'amour sans la raison tourne à l'esclavage, le prophétisme sans l'amour au délire : Isis convient exactement au libéralisme hégémonique. Dans le sein régénérateur de la Déesse Mère, qui, telle les mortes vivantes d'Edgar Poe,

5 De tout ce que l'Église publie officiellement, les médias ne répercutent guère que ce qui touche au sexe. 
remonte de la tombe du paganisme sur laquelle le christianisme n'arrive plus à imposer le couvercle de Dieu le Père, le libéralisme rattrape enfin son retard sur le nazisme en matière de "liberté ": "Honore ton père et ta mère? Ah non. Tout jeune homme se révolte, prend son père en haine, et c'est exactement ce qu'il doit faire pour entrer dans la vie ${ }^{6}$. " Hitler ne voulait-il pas "libérer" les hommes des commandements de Dieu. Au nom de quoi limitera-t-on maintenant la « liberté " du capital, qui voit un obstacle dans toute espèce de loi inspirée par la compassion et l'universalité du droit à la vie. Il y a des jours où je me dis que nos contemporains se trouveront sous peu, plutôt qu'avec Jésus, d'accord avec Gengis Khan : "Le plus grand bonheur est de vaincre ses ennemis, de les pourchasser, de les dépouiller de leurs richesses, de voir en larmes ceux qui leur sont chers, d'étreindre sur sa poitrine leurs femmes et leurs filles. "C'est encore de l'identification à l'autre, pour le plaisir de sentir ce qu'il éprouve quand je l'écrase : cela s'appelle le sadisme et c'est la forme de " compassion" qui domine cette fin de millénaire où l'hystérie redevient un symptôme hurlant de l'impuissance des sensibles.

6 Gobbels cité par Hermann Rauschning, Hitler m'a dit. Paris, Hachetre (Pluriel), 1995. Voir aussi, sur Mein Kampf en tant que « défi insensé lancé par Hitler à Moïse ": Raphaël Draï, La communication prophétique. Tome II, La conscience des prophètes. Paris, Fayard, 1993, pp. 478ss. 